\title{
Tuning Fabry-Perot resonances via diffraction evanescent waves
}

\author{
Bo Hou, ${ }^{1}$ Jun Mei, ${ }^{1}$ Manzhu Ke, ${ }^{2}$ Weijia Wen,,${ }^{1} *$ Zhengyou Liu, ${ }^{2}$ Jing Shi, ${ }^{2}$ and Ping Sheng ${ }^{1}$ \\ ${ }^{1}$ Department of Physics, Hong Kong University of Science and Technology, Clear Water Bay, Kowloon, Hong Kong, China \\ ${ }^{2}$ Department of Physics, Wuhan University, Wuhan 430072, China
}

(Received 22 June 2007; published 14 August 2007)

\begin{abstract}
By studying acoustic and electromagnetic wave transmission through a periodic array of subwavelength holes or slits with various channel lengths, we demonstrate both experimentally and theoretically that diffraction evanescent waves can play an important role in tuning the Fabry-Perot (FP) resonances. In particular, there can be total transmission peaks at wavelengths much below that of the Rayleigh-Wood limit, and FP resonances can occur for channel length $\sim 16 \%$ thinner than the half wavelength. In addition, the FP resonance condition can be tuned via both the periodicity and area fraction of holes. As a function of the ratio between the periodicity and plate thickness, the FP resonance is smoothly linked to the surface-wave-like mode induced by the periodic structure factor.
\end{abstract}

DOI: 10.1103/PhysRevB.76.054303

PACS number(s): 43.35.+d, 43.40.+s, 43.90.+v

\section{INTRODUCTION}

Fabry-Perot (FP) resonance is one of the most basic and common phenomena in both classical and quantum physics. It is a direct manifestation of the constructive or destructive wave interference over a certain path length and can lead to multiple transmission peaks as a function of frequency for wave transmission through impedance-mismatched layers. There is a renewed interest in electromagnetic FP resonances, especially their relevance to the recently observed transmission enhancement through subwavelength openings in normally opaque objects, ${ }^{1-8}$ as well as to the ensuing debate on the relative contributions of surface wave versus diffraction in such phenomena. ${ }^{9-19}$

It has been shown that light transmission through hole arrays in perfect-conductor thin films, where there can be no true surface waves, may be attributed (via Babinet's principle) to the reflection by the complementary structure consisting of planar arrays of perfect-conductor disks. ${ }^{17,18}$ Transmission can always reach unity at some resonant wavelengths regardless of how small the holes are, while the complementary system can exhibit perfect reflection for arbitrarily small disks. These resonances were found to be associated with the divergence in structure factor, owing to the coherent addition of the scatterings from the periodic array of disks or holes. As a result, a quasisurface mode with frequency close to the onset of the first diffraction order (wavelength $\lambda$ slightly larger than the lattice constant $a$ ) always exists. Such modes are denoted "structure-factor-induced surface modes," or SF resonances. As the structure factor mechanism is valid for either the electromagnetic or the acoustic wave, it is expected that such a resonant transmission mechanism also applies to (scalar) acoustic waves. For completeness, we present in the Appendix the relevant arguments for the SF induced resonant transmission for both the electromagnetic and acoustic waves.

In a separate development, it was found that total electromagnetic wave transmission through subwavelength openings can also be associated with FP resonances. Inside the subwavelength openings, either in $\operatorname{slit}^{11-14}$ or annular geometry, ${ }^{15}$ standing wave patterns are formed along the longitudinal direction, giving rise to FP resonances.
The purpose of this work is to show through both theory and experiment that for thick hole arrays, the FP resonances and the SF resonances are in fact the two limits of a single resonance phenomenon, for both the acoustic and electromagnetic waves. Whereas the FP resonance is characterized by constructive interference along the channel direction, the SF-induced resonance is induced by lateral coupling. By varying the plate thickness or channel length, one makes the transition from the FP resonance (thick plate limit) to the SF resonance (thin plate limit). Between the two limits there can be interesting deviation from FP resonance conditions, owing to the interaction of the diffraction evanescent waves. In particular, it is shown that acoustic and electromagnetic FP resonant transmissions can occur not only at frequencies much below the Rayleigh-Wood limit, but also for channel (path) lengths significantly lower than the minimum half wavelength usually required, tunable by varying either the periodicity or the area fraction of holes, or both.

\section{SAMPLES AND MEASUREMENTS}

We first consider ultrasound transmission through a brass plate of thickness $t$, immersed in water. A square lattice array of circular holes with radius $r$ were drilled on the plate, with lattice constant $a>2 r$. A schematic picture of the unit cell is shown as an inset to Fig. 1(a). The ultrasonic transmission measurements in the regime of $0.2-0.8 \mathrm{MHz}$ were done in a large water tank. Two transducers were employed as ultrasonic generator and receiver, and the sample was placed between the two transducers. The ultrasonic beam was normally incident upon the sample and the transmitted signal was collected by the receiver, collinear with the incident wave. The transmission of ultrasonic waves through a smooth brass plate was also measured as the reference. Apart from measuring the far-field transmission, we have also implemented near-field scanning to identify the role of surface waves, if any, in the transmission process. A pinducer with a diameter of $1.5 \mathrm{~mm}$ was mounted on a twodimensional translation stage and brought to a distance, $z$, from the rear surface of the sample to detect the surface field distribution. Scanning was done along the $x-y$ plane parallel 

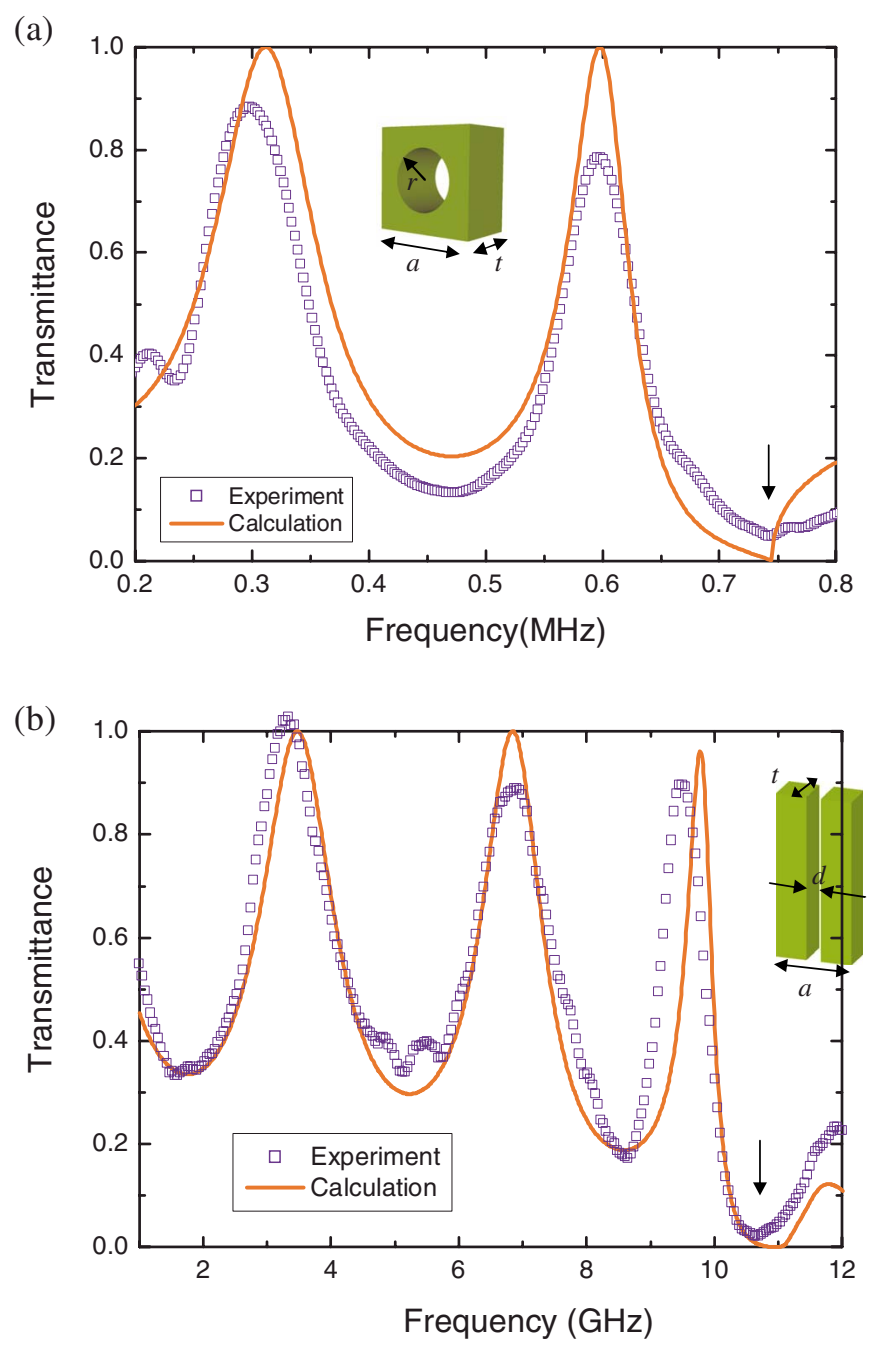

FIG. 1. (Color online) (a) Transmittance of an ultrasonic wave at normal incidence through the structured brass plate with $a$ $=2.0 \mathrm{~mm}, r=0.6 \mathrm{~mm}$, and $t=2.0 \mathrm{~mm}$. (b) Transmittance of microwave at normal incidence through the metallic grating of onedimensional slits with periodicity $a=28 \mathrm{~mm}$, slit width $d=9 \mathrm{~mm}$, and thickness $t=38 \mathrm{~mm}$. The transmission peak being $>100 \%$ is due to the finite size effect of the sample. The arrow indicates the Rayleigh-Wood frequency. Insets: Schematic pictures of the unit cells for the acoustic and electromagnetic samples.

to the brass plate surface, with a spatial resolution of 0.1 $\times 0.1 \mathrm{~mm}^{2}$.

For the electromagnetic (EM) case, we consider microwave transmission through a metallic grating constructed by stacking aluminum-alloy slabs with spacers at both ends. A schematic picture of the unit cell structure is shown as an inset to Fig. 1(b). A microwave of $1-12 \mathrm{GHz}$ emitted from a horn antenna was normally incident with the electrical field perpendicular to the slits. By employing different-sized slabs we measured three cases of periodicity-to-thickness ratio $=0.74,1.48$, and 2.95 , with the area fraction of slits maintained at $32 \%$.

There is a difference between the acoustic FP resonance from the electromagnetic case, owing to the existence of the scalar channel that allows the propagation of an acoustic wave through holes whose transverse dimensions are subwavelength. ${ }^{20}$ The simplified cases of a thin screen, ${ }^{21,22}$ a single circular channel, ${ }^{23,24}$ and one-dimensional slits ${ }^{25}$ have received extensive prior attention. Specifically, we consider here the acoustic transmission in the case of very long channels and found FP-like multiple transmission peaks appearing at wavelengths much below the diffraction limit, noted to differ from that studied in Ref. 25.

In Fig. 1(a) we show the measured acoustic wave transmission as a function of frequency. It is seen that there can be multiple transmission peaks well below the RayleighWood frequency (the diffraction limit, indicated by the arrow, corresponds to the wavelength $\lambda=a$ at normal incidence). A smooth brass plate of thickness $2.0 \mathrm{~mm}$ has a very low transmittance of $<10 \%$ within the measured frequency regime, whereas for the structured brass plate two peaks at 0.310 and $0.598 \mathrm{MHz}$ were observed, in good agreement with the calculations to be described below. However, the peak frequencies display no direct correlation with the FP resonance condition. In Fig. 1(b), the EM transmission data are shown. Three transmission peaks are seen. They likewise do not correspond with the usual FP resonance condition.

\section{THEORETICAL MODEL}

In order to establish a useful model, we first note that the relevant ultrasonic frequencies $\omega \gg 2 \pi / \tau$, where $\tau=\rho_{f} r^{2} / \eta$ $\cong 1 \mathrm{~s}$ is the viscous relaxation time for water density $\rho_{f}$ $=1 \mathrm{~g} / \mathrm{cm}^{3}$, viscosity $\eta=0.01 \mathrm{P}$, and $r \cong 0.1 \mathrm{~cm}$. That implies for the relevant frequencies the inertial force density is much larger, and for the purpose of considering wave propagation the fluid may be regarded as inviscid. We next consider the relevance of a Stoneley ${ }^{26}$ wave (the elastic wave equivalent to surface plasmon, bound and propagates along the fluidsolid interface) to the present phenomenon, by directly measuring the wave intensity as a function of distance $z$ away from the brass plate surface. By placing the pinducer at distances $z=0.02$ and $0.17 \mathrm{~mm}$, the scanned acoustic wave intensities at the transmission peak frequency of $0.598 \mathrm{MHz}$ are shown in Figs. 2(a) and 2(c), respectively. By considering the plate as rigid (thus not allowing surface Stoneley waves), the calculated intensity patterns at the same distances and frequency are also shown in Figs. 2(b) and 2(d). The agreement is seen to be excellent, implying that the surface wave plays no role in the present transmission phenomenon.

For the EM case, there cannot be a true surface plasmon, as there cannot be any tangential electric field on the metallic surface in the microwave regime. ${ }^{27}$ Nevertheless, due to the existence of slits, there can be surface-wave-like behavior induced by the lateral structural factor. ${ }^{17,18}$ The absence of a surface Stoneley wave and surface plasmon simplifies the transmission mechanism and implies that both acoustic and EM phenomena may fall under a unified framework. The theory and measured transmission data for both, as a function of inverse plate thickness, are thus plotted in Figs. 3 and 4.

Consider the Helmholtz equation,

$$
\nabla^{2} \Phi+\left(\omega^{2} / c^{2}\right) \Phi=0 .
$$

For the acoustic wave, $c=1490 \mathrm{~m} \mathrm{~s}^{-1}$ and $\Phi$ is the scalar 
velocity potential. For the EM case, $c=3.0 \times 10^{8} \mathrm{~m} \mathrm{~s}^{-1}$ and $\Phi=H_{y}$ (the component parallel to the slit). From $\Phi$ the local displacement velocity is obtained as $\vec{v}=\nabla \Phi$ and pressure $p$ $\propto \partial \Phi / \partial t$. Given the magnetic field, the electric field is given by $\vec{E}=(i / \omega \varepsilon) \nabla \times \vec{H}$. The potential can be expanded in terms of the waveguide modes inside the hole [Eq. 2a) for the acoustic wave case] or slit [Eq. (2b) for the EM wave case] as

$$
\Phi_{1}=\left\{\begin{array}{l}
\sum_{n} J_{0}\left(\alpha_{n} \rho\right)\left[A_{n} \exp \left(-\beta_{n} z\right)+B_{n} \exp \left(\beta_{n} z\right)\right], \\
\sum_{n} \cos [(n \pi / d)(x+d / 2)]\left[E_{n} \exp \left(-i \gamma_{n} z\right)+F_{n} \exp \left(i \gamma_{n} z\right)\right] .
\end{array}\right.
$$

In Eq. (2a), $\alpha_{n}^{2}-\beta_{n}^{2}=\omega^{2} / c^{2},\left.\frac{d J_{0}\left(\alpha_{n} \rho\right)}{d \rho}\right|_{\rho=r}=0$, where $r$ is the radius of each hole, $J_{0}$ denotes the zeroth order Bessel function, $\rho$ is the transverse radial coordinate, and $\alpha_{n}=x_{n} / r$ is the radial wave vector, with $x_{n}$ being the $n$th zero of $J_{0}$. In Eq. $(2 \mathrm{~b}), \gamma_{n}$ $=\sqrt{(\omega / c)^{2}-(n \pi / d)^{2}}, d$ is the width of each slit. The potentials in front of and behind the plate are expanded, respectively, as

$$
\Phi_{2}=\left\{\begin{array}{l}
P \exp (i \omega z / c)+\sum_{\vec{G}} C_{G} \exp (i \vec{G} \cdot \vec{\rho}) \exp \left[-i \sqrt{(\omega / c)^{2}-G^{2}} z\right], \\
P \exp (i \omega z / c)+\sum_{m} K_{m} \exp (i 2 m \pi x / a) \exp \left[-i \sqrt{(\omega / c)^{2}-(2 m \pi / a)^{2}} z\right],
\end{array}\right.
$$

and

$$
\Phi_{3}=\left\{\begin{array}{l}
\sum_{\vec{G}} D_{G}^{-} \exp (i \vec{G} \cdot \vec{\rho}) \exp \left[i \sqrt{(\omega / c)^{2}-G^{2}} z\right], \\
\sum_{m} L_{m} \exp (i 2 m \pi x / a) \exp \left[i \sqrt{(\omega / c)^{2}-(2 m \pi / a)^{2}} z\right]
\end{array}\right.
$$

(a)

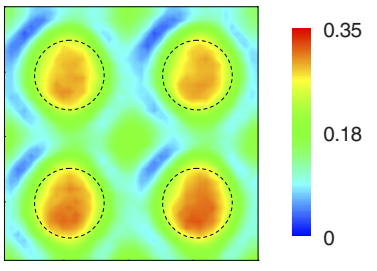

Experiment

(c)

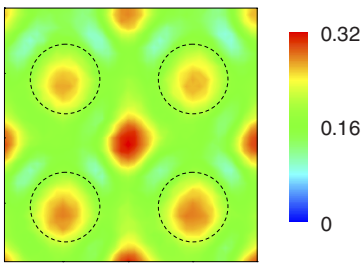

Experiment

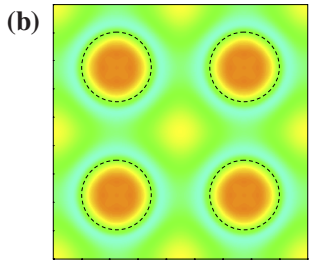

Theory

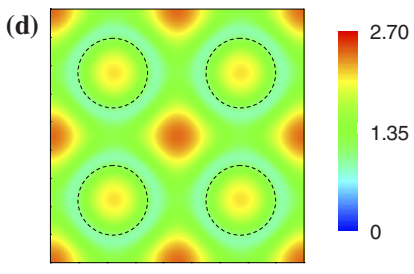

Theory

FIG. 2. (Color online) Intensity distributions of pressure field at the rear surface of the sample for two selected distances, $z=0.02$ and $0.17 \mathrm{~mm}$, at the resonance frequency of $0.598 \mathrm{MHz}$. The sample has the same parameter values as those in Fig. 1. (a) and (b) are the measured and calculated field pattern at $z=0.02 \mathrm{~mm}$, respectively; (c) and (d) are the same for $z=0.17 \mathrm{~mm}$, respectively. The holes are delineated by dashed lines.

where the normally incident wave amplitude is denoted by $P$, $\vec{G}=n_{1} \frac{2 \pi}{a} \hat{x}+n_{2} \frac{2 \pi}{a} \hat{y}$ with $n_{1}$ and $n_{2}$ being integers, and $a$ denoting the periodicity for both the hole arrays (in the sonic case) and of the metallic grating (in the microwave case). The oblique incidence case can be similarly treated by modifying the expansions for $\Phi_{2}$ and $\Phi_{3}$. By requiring continuity of $v_{z}$ and $p$ at the interfaces between $\Phi_{1}, \Phi_{2}$, and $\Phi_{3}$ over the hole region, vanishing $v_{z}$ elsewhere on the interfaces for the acoustic wave, by requiring the continuity of $H_{y}$ and $E_{x}$ at the surfaces of the grating over the slit region, and vanishing $E_{x}$ elsewhere on the interfaces, we obtain the necessary equations for the solution of reflection and transmission coefficients. Resonance modes are obtained by setting $P=0$. It can be seen from Fig. 3 that the EM wave transmission frequencies for a metallic gratings with $d / a=0.32$ have identical behavior as the acoustic case for a hole array with $r / a$ $=0.30$.

The predictions of the theory, as well as the experimental results, are shown in Figs. 1(a) and 1(b). It is seen that with no adjustable parameters, the agreement is very good, especially in the positions of the transmission peaks. For the EM case, the solid line in this case is obtained from the finitedifference time-domain simulation. ${ }^{28}$ 


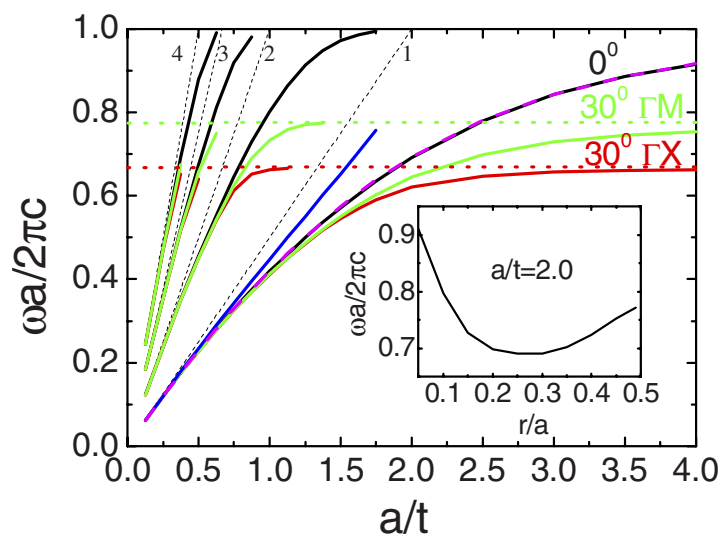

FIG. 3. (Color online) Calculated acoustic and EM transmission frequencies for a square lattice of holes and metallic grating, respectively, plotted as a function of inverse channel length. Black solid lines are for normal incidence, whereas the green and red lines are for incident angle $\theta=30^{\circ}$ along the $[1,1]$ and $[0,1]$ directions, respectively. Green $\left(30^{\circ} \mathrm{\Gamma M}\right)$ and red $\left(30^{\circ} \mathrm{\Gamma X}\right)$ dashed lines indicate the Rayleigh-Wood frequencies along these two directions. Black dashed lines delineate the Fabry-Perot condition, $t=n \lambda / 2$, with the order $n$ denoted. The magenta dashed line represents the EM wave transmission peaks at normal incidence for a metallic grating with $d / a=0.32$. The blue line is determined by Eq. (5), with a slope of $\sim 0.42$ (i.e., $t \cong 0.42 \lambda$ ). Inset: Calculated resonant transmission frequencies for a plate with a square lattice of holes, in which $a=2.0 \mathrm{~mm}, t=1.0 \mathrm{~mm}$, and $r$ is varied from 0.1 to $1 \mathrm{~mm}$. A minimum occurs at $r=0.3 a$.

\section{CHARACTERISTICS OF THE TRANSMISSION RESONANCES}

We wish to show that the observed transmission peaks are the manifestation of a type of resonance mode that has FP and SF resonances as the two limits. The diffraction evanescent modes play an important role in interpolating between the two limits. To make explicit the role of diffraction evanescent modes, we resort to analyzing the acoustic problem by retaining only one cylindrical mode $\left[J_{0}\left(\alpha_{1} \rho\right)=J_{0}(0)=1\right]$ and five plane wave modes $\left[\vec{G}=(0,0)\right.$ and $\left.\vec{G}=( \pm 1, \pm 1) \frac{2 \pi}{a}\right]$, both in $\Phi_{2}$ and $\Phi_{3}$. In principle, by keeping more cylindrical modes and more plane wave modes, the results obtained should be more accurate, but they do not add to the physical understating. With the minimal number of modes, the resulting resonant mode equation for the acoustic case can be expressed as

$$
\tan (2 t \omega / c)=\frac{-a^{3} k_{0} k_{1}^{2}}{\frac{\pi\left(1-f^{2}\right)}{16 J_{1}^{2}(2 \pi r / a)} a^{3} k_{1}^{3}+\pi^{2} \frac{2 a}{t},}
$$

in which $k_{0}=\omega / c, k_{1}=\sqrt{(2 \pi / a)^{2}-(\omega / c)^{2}}$ is the diffraction evanescent wave vector, $f$ denotes the area fraction of holes, and $J_{1}$ is the first order Bessel function. Equation (5) is instructive, since a vanishing right-hand side would directly yield the FP resonance condition, $t=n \lambda / 2, \lambda$ being the wavelength. A combination of hole and periodic diffraction eva- nescent wave effects constitute the correction to the usual FP condition in the form of a nonzero right-hand side, implying that the FP resonance can be tuned by varying the periodicity and area fraction of holes. We denote such resonances the FPEV resonances (EV means evanescent wave).

In Fig. 3 we show the FPEV resonance frequencies plotted as a function of inverse plate thickness. The FP condition is indicated by the black dashed straight lines, for $n=1-4$, with slopes of $0.5,1,1.5$, and 2, respectively. The FPEV frequencies are shown as solid black (normal incidence) and red and green $\left(30^{\circ}\right.$ oblique incidence) lines. Compared with the FP resonances, it is seen that the FPEV resonances always occur at lower frequencies, as though the effective plate thickness is greater than $t$. The prediction of Eq. (5), for the $n=1 \mathrm{FPEV}$ resonance, is shown as the blue line. Except in the region of very small $a / t$ values, the blue line has a slope of 0.42 . Thus the effect of the diffraction evanescent waves is to shift the resonance condition by $\sim 16 \%$, in the direction of smaller channel length. The difference between the prediction of Eq. (5) and the red and green lines appears at the small $t$ limit, where the transmission peak frequency shows a clear dependence on the incidence angle. This is characteristic of the surface-wave-like mode induced by the SF resonance. ${ }^{17,18}$ In fact, these transmission peak frequencies all occur at close to the Rayleigh-Wood limit, as required by the SF resonance condition. Thus the lowest frequency FPEV resonance, which shows little or no dependence on the incidence angle, is smoothly converted to the structure-factor-induced surface mode in the thin-plate limit. The diffraction evanescent wave contributions are dominant at the intermediate values of $a / t$. To a lesser degree, similar behavior can be observed for the higher order FPEVs.

In the same figure we also show (the magenta line) the prediction of the EM wave case for a metallic grating. The fact that the acoustic and EM curves are almost identical implies a unified framework for the underlying mechanism, as expected.

It is seen that as the ratio $a / t$ increases, the lowest order evanescent waves [Eq. (5)] can no longer account for the resonant frequency trajectory. Also, in the large $a / t$ limit the curves also display pronounced incident angle dependence, in contrast to FP resonances, which are nearly independent of the incidence angle. These are the signals for (1) the lateral scattering interaction is contributing much more to the resonant modes, hence the lowest order evanescent modes are no longer sufficient to account for such strong lateral interactions, and (2) with the increased lateral interaction, the SF effect becomes more pronounced, implying incidence angle dependence as shown in the Appendix.

The inset to Fig. 3 shows the FPEV's frequency dependence (lowest frequency mode) on $r / a$ for the acoustic case. There is a clear minimum, indicating a competition between the single-hole evanescent wave diffraction effect and the influence of periodic array on a single hole. Only when the two effects are constructively combined, the biggest deviation from the usual FP resonant condition is achieved, as shown in the inset.

We have also measured the acoustic samples with various plate thicknesses ranging from 0.5 to $3.1 \mathrm{~mm}$. Figure 4 sum- 


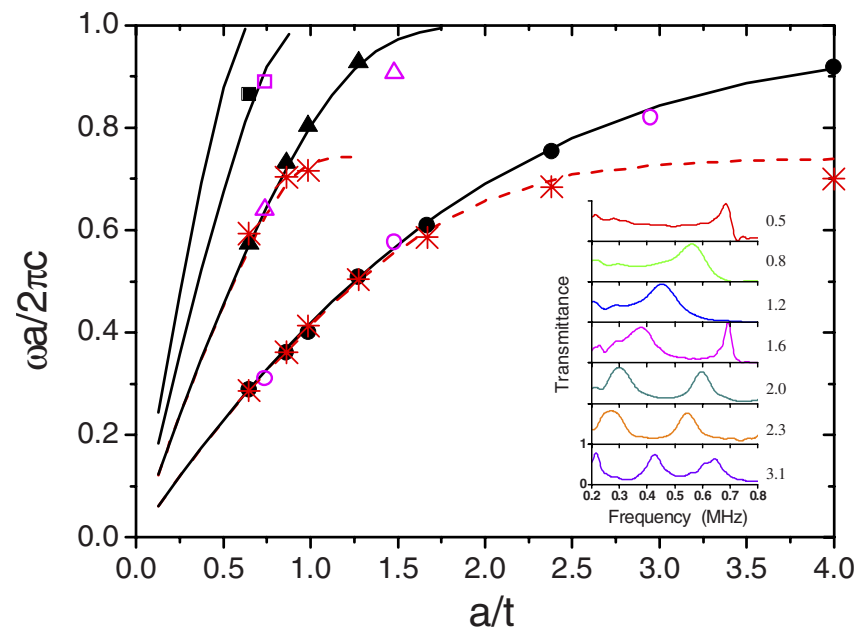

FIG. 4. (Color online) Solid symbols and solid lines are the measured and calculated resonant transmission frequencies of a normally incident acoustic wave on a square lattice of holes with $r$ $=0.3 a$, respectively. The solid lines are the same as the black solid lines in Fig. 3. Star symbols (measurement) and dashed lines (calculation) denote the case of an acoustic wave at $20^{\circ}$ incidence angle along the $[0,1]$ direction. Open symbols represent the measured transmission peaks of a normally incident microwave on a metallic grating with $d / a=0.32$. The inset shows the measured acoustic transmittances at normal incidence for various plate thicknesses, with $a=2.0 \mathrm{~mm}$ and $r=0.6 \mathrm{~mm}$. The thickness (in $\mathrm{mm}$ ) of each plate is given to the right of the spectrum.

marizes all the measured transmission peak frequencies for the acoustic and the EM cases and their comparison with theory. Excellent agreement is seen. In particular, the experimental data demonstrate the validity of the scaling relation

$$
\frac{\omega_{n}}{2 \pi c / a}=g_{n}\left(\frac{a}{t}, \hat{k}_{\mathrm{inc}}\right)
$$

where $\omega_{n}$ is the FPEV resonance frequency, the subscript $n$ denotes the order of the FPEV resonances, $\hat{k}_{\text {inc }}$ denotes the unit vector along the incident direction, and $g_{n}$ represents a function with two arguments. Equation (6) demonstrates that the FPEV resonance frequencies for the hole array depend on three nontrivial variables: the ratio $a / t$, the incidence direction, and the order of the resonance $n$. The ratio $r / a$ (or the area ratio of the openings) can slightly adjust the frequencies in the intermediate $a / t$ regime.

\section{CONCLUDING REMARKS}

We have shown that both acoustic and electromagnetic waves share the common resonant transmission phenomenon, with FP and SF induced resonances as the two limits. Generalization of the basic ideas presented in this work may offer experimental tests to the role of diffraction evanescent waves in the subwavelength optical transmission phenomenon.

\section{ACKNOWLEDGMENT}

This work was supported by NSFC/RGC joint research grants No. HKUST605/04, No. 10418014, and No. HKUST3/06C.

\section{APPENDIX}

According to Babinet's principle, light transmission through a hole array in perfect-conductor thin film is related to the reflectance of the complementary structure consisting of a planar array of perfect-conductor thin disks. Hence to demonstrate SF-induced resonance mechanism for perfect transmission through hole arrays, it is only necessary to demonstrate the perfect reflection of its complementary structure. By following the approach that has been given by García de Abajo et al., ${ }^{17,18}$ this can be obtained self-consistently by solving for the electric dipoles induced on the disks,

$$
\vec{p}_{R}=\alpha_{E} \vec{E}^{\mathrm{inc}}(\vec{R})+\alpha_{E} \sum_{\vec{R}^{\prime} \neq \vec{R}} G_{E}^{\overrightarrow{R R^{\prime}}} \vec{p}_{\vec{R}^{\prime}},
$$

where $\vec{R}$ and $\vec{R}^{\prime}$ label disk sites, $\alpha_{E}$ is the electric polarizability of the disks, and $G_{E}^{R R^{\prime}}$ is the dipole-dipole interaction dyadic. ${ }^{17}$ At normal incidence on periodic arrays, the polarization is the same for all disks, given by

$$
p=\frac{1}{\frac{1}{\alpha_{E}}-G_{E}},
$$

where

$$
G_{E}=\sum_{\substack{R \neq 0\\}}\left(k^{2}+\partial_{x x}^{2}\right) e^{i k R} / R .
$$

It follows that the reflectance of the disk array, which equals the transmittance of the complementary hole array, is given by

$$
T=\frac{1}{1+\left(\frac{A}{2 \pi k} \operatorname{Re}\left\{\alpha_{E}^{-1}-G_{E}\right\}\right)^{2}},
$$

where $A$ is the area of the unit cell.

The structure factor $G_{E}$ diverges when one of the reflected beams goes grazing, and in particular near $\lambda=a$, the period of the square array. As shown in Ref. 17, $\operatorname{Re}\left\{G_{E} a^{3}\right\}$ $\approx 4 \pi^{2} \sqrt{2} / \sqrt{\lambda / a-1}+35 \exp [-22(\lambda / a-1)]-118$. Obviously, $G_{E}$ diverges as $\lambda \rightarrow a^{+}$. Thus even for an arbitrarily small hole diameter, there is always one wavelength $\lambda>a$ for which the second term in the denominator of Eq. (A4) vanishes, implying perfect transmittance (reflectance) at that frequency for the arrays of holes (disks). Evidently, this divergence of $G_{E}$ is closely related to the structure factor of the system.

For (scalar) acoustic waves, a similar conclusion can be made. Evidently, it is more convenient mathematically to deal with a square array of spheres instead of a square array of disks, with the underlying physics being the same. It was 
shown ${ }^{29}$ that the far-field scattering amplitude of a soundsoft sphere (with radius $a$ ) induced by a plane incident wave can be written as $-a \frac{e^{i k r}}{r}$ in the small sphere limit of $k a \ll 1$. Thus an "acoustic polarizability" $\alpha=-a$ can be similarly defined by self-consistently writing down the following equation:

$$
p_{R}^{-}=\alpha I(\vec{R})+\alpha \sum_{\overrightarrow{R^{\prime}} \neq \vec{R}} G_{R R^{\prime}}^{-\vec{p}}-\overrightarrow{R^{\prime}},
$$

where $\vec{R}$ and $\vec{R}^{\prime}$ label the sphere sites, $p_{R}$ is the secondary source strength at position $R, I(R)$ denotes the incident acoustic field, and $G_{R R^{\prime}}$ is the acoustic field at $\vec{R}$ induced by the scattered field of the sphere at $\vec{R}^{\prime}$. At normal incidence on periodic arrays, the "acoustic polarization" is the same for all spheres, given by

$$
p=\frac{1}{\frac{1}{\alpha}-G} I,
$$

where

$$
G=\sum_{\vec{R} \neq 0} e^{i k R} / R
$$

Just like the EM wave case, $G$ diverges as $\lambda \rightarrow a^{+}$. Thus, given an arbitrarily small sphere radius $a$, there is always one wavelength $\lambda>a$ for which the denominator of Eq. (A6) vanishes, implying the coherent interaction of all the spheres, leading to the divergence of the acoustic impedance of the square array of the spheres. At this resonant frequency, the reflection from the sphere or disk array would be $100 \%$ due to the divergence of its impedance. From Babinet's principle, acoustic transmission through the hole arrays in a thin rigid slab can reach $100 \%$ due to the SF mechanism.

It should be noted that for the SF induced mechanism, the resonance frequency can vary with the incidence angle. When the light (or sound) wave is obliquely incident with an incident angle $\theta_{i}$, the divergence frequency $\omega_{d}$ of the structure factor $G$ should be determined by the condition that the first-order diffraction beam becomes tangent to the plane of grating, i.e., $\omega_{d}=2 \pi c /\left[a\left(1+\sin \theta_{i}\right)\right]$. It follows that the resonant transmission frequency is also dependent on the incident angle since the resonant frequency is slightly smaller than the divergence frequency $\omega_{d}$.
*Corresponding author. phwen@ust.hk

${ }^{1}$ T. W. Ebbesen, H. J. Lezec, H. F. Ghaemi, T. Thio, and P. A. Wolff, Nature (London) 391, 667 (1998).

${ }^{2}$ H. F. Ghaemi, T. Thio, D. E. Grupp, T. W. Ebbesen, and H. J. Lezec, Phys. Rev. B 58, 6779 (1998).

${ }^{3}$ D. E. Grupp, H. J. Lezec, T. W. Ebbesen, K. M. Pellerin, and T. Thio, Appl. Phys. Lett. 77, 1569 (2000).

${ }^{4}$ A. Krishnan, T. Thio, T. J. Kim, H. J. Lezec, T. W. Ebbesen, P. A. Wolff, J. Pendry, L. Martin-Moreno, and F. J. Garcia-Vidal, Opt. Commun. 200, 1 (2001).

${ }^{5}$ L. Martín-Moreno, F. J. García-Vidal, H. J. Lezec, K. M. Pellerin, T. Thio, J. B. Pendry, and T. W. Ebbesen, Phys. Rev. Lett. 86, 1114 (2001).

${ }^{6}$ W. L. Barnes, W. A. Murray, J. Dintinger, E. Devaux, and T. W. Ebbesen, Phys. Rev. Lett. 92, 107401 (2004).

${ }^{7}$ L. Martín-Moreno and F. J. García-Vidal, Opt. Express 12, 3619 (2004).

${ }^{8}$ W. L. Barnes, A. Dereux, and T. W. Ebbesen, Nature (London) 424, 824 (2003).

${ }^{9}$ M. M. J. Treacy, Phys. Rev. B 66, 195105 (2002).

${ }^{10}$ H. J. Lezec and T. Thio, Opt. Express 12, 3629 (2004).

${ }^{11}$ J. A. Porto, F. J. García-Vidal, and J. B. Pendry, Phys. Rev. Lett. 83, 2845 (1999).

${ }^{12}$ H. E. Went, A. P. Hibbins, J. R. Sambles, C. R. Lawrence, and A. P. Crick, Appl. Phys. Lett. 77, 2789 (2000).

${ }^{13}$ Y. Takakura, Phys. Rev. Lett. 86, 5601 (2001).

${ }^{14}$ F. Yang and J. R. Sambles, Phys. Rev. Lett. 89, 063901 (2002).

${ }^{15}$ F. I. Baida, D. V. Labeke, G. Granet, A. Moreau, and A. Belkhir, Appl. Phys. B: Lasers Opt. 79, 1 (2004).

${ }^{16}$ D. C. Skigin and R. A. Depine, Phys. Rev. Lett. 95, 217402
(2005).

${ }^{17}$ F. J. García de Abajo, R. Gómez-Medina, and J. J. Sáenz, Phys. Rev. E 72, 016608 (2005).

${ }^{18}$ F. J. García de Abajo and J. J. Sáenz, Phys. Rev. Lett. 95, 233901 (2005).

${ }^{19}$ S. Selcuk, K. Woo, D. B. Tanner, A. F. Hebard, A. G. Borisov, and S. V. Shabanov, Phys. Rev. Lett. 97, 067403 (2006).

${ }^{20}$ The condition of normal velocity must be zero at the channel wall implies that it is the derivative of $J_{0}\left(=-J_{1}\right)$ which must be zero at the solid boundary. However, $J_{1}(0)=0$, implying no cutoff for the acoustic wave propagation in subwavelength holes. See, L. D. Landau and E. M. Lifshitz, Fluid Mechanics, 2nd ed. (Pergamon Press, New York, 1987).

${ }^{21}$ H. Lamb, Hydrodynamics, 6th ed. (Dover, New York, 1991).

${ }^{22}$ J. Miles, Wave Motion 35, 311 (2002).

${ }^{23}$ G. P. Wilson and W. W. Soroka, J. Acoust. Soc. Am. 37, 286 (1965).

${ }^{24}$ K. H. Jun and H. J. Eom, J. Acoust. Soc. Am. 98, 2324 (1995).

${ }^{25}$ X. Zhang, Phys. Rev. B 71, 241102(R) (2005).

${ }^{26}$ R. Stoneley, Proc. R. Soc. London, Ser. A 106, 416 (1924).

${ }^{27}$ B. Hou, Z. H. Hang, W. Wen, C. T. Chan, and P. Sheng, Appl. Phys. Lett. 89, 131917 (2006).

${ }^{28}$ Simulations were implemented by using commercial software CONCERTO 3.5 (Vector Fields Limited, England, 2003). In the simulation, a periodical boundary condition was imposed to the unit cell shown as the inset of Fig. 1(b), and a perfect conductor approximation was adopted.

${ }^{29}$ Paul Martin, Multiple Scattering (Cambridge University Press, Cambridge, England, 2006). 